\title{
Food allergies and food-induced anaphylaxis: role of cofactors
}

Meeyong Shin, MD, PhD

Department of Pediatrics, Soonchunhyang University Bucheon Hospital, Soonchunhyang University School of Medicine, Bucheon, Korea

Food allergies and food-induced anaphylaxis are important health problems. Several cofactors modulating the onset of anaphylaxis have been identified. In the presence of cofactors, allergic reactions may be induced at lower doses of food allergens and/or become severe. Exercise and concomitant infections are well-documented cofactors of anaphylaxis in children. Other factors such as consumption of nonsteroidal anti-inflammatory drugs, alcohol ingestion, and stress have been reported. Cofactors reportedly play a role in approximately $30 \%$ of anaphylactic reactions in adults and $14 \%-18.3 \%$ in children. Food-dependent exercise-induced anaphylaxis (FDEIA) is the best-studied model of cofactor-induced anaphylaxis. Wheatdependent exercise-induced anaphylaxis, the most common FDEIA condition, has been studied the most. The mechanisms of action of cofactors have not yet been fully identified. This review aims to educate clinicians on recent developments in the role of cofactors and highlight the importance of recognizing cofactors in food allergies and food-induced anaphylaxis.

Key words: Food hypersensitivity, Anaphylaxis, Cofactors

\section{Key message}

Question: What are the roles of cofactors in food allergies and food-induced anaphylaxis?

Finding: Cofactors reportedly play a role in approximately $14 \%-30 \%$ of anaphylactic reactions. Cofactors such as exercise, infection, nonsteroidal anti-inflammatory drugs, dehydration, and alcohol can increase intestinal permeability and antigen uptake, thereby causing allergic symptoms.

Meaning: Routine assessment of the possible involvement of cofactors is essential for the management of patients with foodinduced anaphylaxis.

\section{Introduction}

Food allergies are an important health problem and the most common cause of anaphylaxis in children. ${ }^{1,2)}$ In clinical practice, physicians encounter patients allergic to food with symptoms only in particular situations. Cofactors and augmenting factors may explain why anaphylaxis can occur with food allergen exposure that can be tolerated or elicit only a mild reaction. However, there is still a lack of evidence regarding the real role of cofactors.

The symptom development of food allergies is thought to be modified by everyday lifestyle factors such as exercise, sleep, and infection. The amount of food that elicits the symptoms varies among patients, especially with different health states. To manage food allergies and anaphylaxis, it is important to know the cofactors affecting a patient's symptoms and the threshold dose of offensive food. In the case of a suggestive history but a negative challenge, the possible involvement of cofactors should be considered. In this situation, oral food challenges combined with cofactors may be helpful.

Cofactors are defined as patient-related or external circumstances associated with more severe allergic reactions. ${ }^{3)}$ Niggemann and Beyer ${ }^{4}$ distinguish the augmenting factors from cofactors depending on their influencing mechanisms: augmenting factors, which influence the immunological mechanism of allergy, such as physical exercise, acute infections, drugs, alcohol, and menstruation, and cofactors, defined as a subgroup of risk factors not acting on an immunological basis themselves, such as certain stages of development (e.g., adolescence), and psychological factors (e.g., emotional stress). However, their underlying mechanisms have not been fully identified; therefore, the term cofactor will be used in this review.

The main cofactors in children and adolescents differ from those in adults. ${ }^{5)}$ In adults, drugs and alcohol are the most frequent cofactors, followed by physical exercise. In contrast, exercise and concomitant infections were the most frequent cofactors of anaphylaxis in children and adolescents. Other factors such as stress, menstruation, and unfavorable climatic conditions have been reported. ${ }^{6,7)}$ The most common cofactors in patients with foodinduced anaphylaxis are shown in Table 1.

Cofactors reportedly play a role in approximately $30 \%$ of anaphylactic reactions in adults ${ }^{7,8)}$ versus $14 \%-18.3 \%$ in children. $5,8,9)$ In one study, at least one cofactor was reported in $40 \%$ of children with anaphylactic reactions. ${ }^{10)}$ Most evidence on cofactors arose from studies in adult patients; thus, the role of cofactors has not yet been optimally studied in children. Moreover, there is limited evidence on how often cofactors play a role

Corresponding author: Meeyong Shin, MD, PhD, Department of Pediatrics, Soonchunhyang University Bucheon Hospital, Soonchunhyang University School of Medicine, 170 Jomaru-ro, Bucheon 14584, Korea 


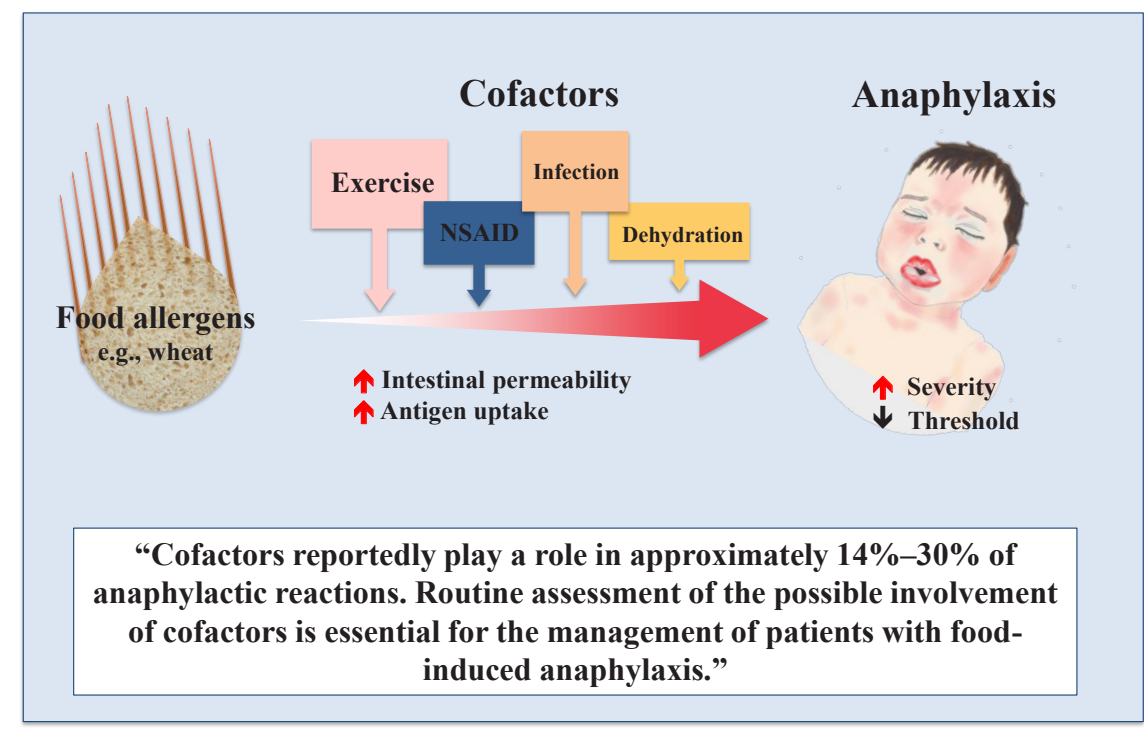

Graphic abstract. The roles of cofactors in food allergies and food-induced anaphylaxis

Table 1. Common cofactors of food-induced anaphylaxis (in order)

Children/adolescents
1. Physical exercise
2. Infection
3. Drugs
4. Stress
5. Others (sleep deprivation, dehydration, menstruation)
Adult
1. Drugs
2. Alcohol
3. Physical exercise
4. Stress
5. Infection
6. Others (sleep deprivation, dehydration, menstruation)

in food-allergic reactions. The frequency of cofactor-implicated anaphylaxis and the role of each cofactor in children and adolescents vary among studies, and a wide range in the frequency of each cofactor has been reported in food-allergic reactions (Table 2). When tiredness and stress are included, in $74 \%$ of the reactions, $\geq 1$ potential cofactors were reportedly present, including tiredness (38\%), alcohol intake (16\%), stress (14\%), sickness/flu (3\%), physical exercise (3\%), and use of analgesics (2\%). ${ }^{11)}$ In a study on patients $\geq 16$ years of age with food allergies, $13 \%$ reported more severe symptoms to food after the involvement of one or more of the following cofactors: physical exercise (10\%), alcohol consumption (5\%), and use of analgesics $(0.6 \%){ }^{12)}$

Food-dependent exercise-induced anaphylaxis (FDEIA) is the best-studied model of cofactor-induced anaphylaxis. In a study of 74 adult cases of suspected cofactor-enhanced food allergies, anaphylaxis accounted for $85.1 \%$ of reactions. ${ }^{6}$ Wheat-dependent exercise-induced anaphylaxis (WDEIA), the most common condition of FDEIA, has been studied the most.

No well-designed prospective study to date has investigated the effects of cofactors on food allergies. A recent study prospectively investigated the effects of cofactors and showed that exercise and sleep deprivation substantially reduced the threshold of reactivity in patients with peanut allergy, putting them at greater risk of a reaction. ${ }^{13)}$

Two main effects of cofactors on food-allergic reactions have been postulated: (1) decreasing threshold; and (2) increasing severity. ${ }^{4)}$ The mechanisms of cofactors involved in allergic reactions are complex and have diverse pathways (Table 3). The mechanisms of action of such cofactors have not been fully identified yet, not even in WDEIA. Two major mechanisms of cofactor-induced modulation eliciting anaphylaxis have been indicated: increased bioavailability of the allergen (increased intestinal permeability) and decreased activation threshold at the cellular level. ${ }^{7,14)}$ In some patients, more than one cofactor may be required to induce anaphylaxis, such as physical exercise and nonsteroidal anti-inflammatory drug (NSAID) use or exercise and alcohol consumption. ${ }^{15,16)}$

This review aims to provide an update for clinicians on the recent developments in the role of cofactors and highlight the importance of recognizing cofactors in food allergies and foodinduced anaphylaxis.

\section{Exercise}

Physical exercise is the most frequently reported cofactor in children and adults. $\left.{ }^{9}{ }^{12}\right)$ In fact, exercise reportedly plays a role as a cofactor in approximately 3\%-10\% of anaphylactic reactions. A recent study investigated how often cofactors play a role in patients aged $\geq 16$ years with food allergies. Of 496 patients with food allergies, $10 \%$ reported physical exercise as a cofactor within 2 hours after consumption of the suspected food. ${ }^{12)}$ Patients with a mild or moderate food allergy reported substantially less frequent involvement of cofactors compared to patients 
Table 2. Frequency of cofactor-implicated anaphylaxis in children and adolescents

\begin{tabular}{|c|c|c|c|c|}
\hline \multirow{2}{*}{ Variable } & \multicolumn{4}{|c|}{ Study } \\
\hline & Uguz et al. ${ }^{8)}$ & Hompes et al. ${ }^{9)}$ & Worm et al. ${ }^{5)}$ & Oropeza et al. ${ }^{10)}$ \\
\hline Year & $2000-2001$ & $2005-2008$ & 2006-2009 & $2013-2014$ \\
\hline Subpopulation & $\begin{array}{l}\text { Anaphylaxis (Foods are } \\
\text { implicated in 89\%.) }\end{array}$ & $\begin{array}{l}\text { Anaphylaxis (Foods are } \\
\text { implicated in 58\%.) }\end{array}$ & Food-induced anaphylaxis & $\begin{array}{c}\text { Anaphylaxis (Foods are } \\
\text { implicated in 65\%.) }\end{array}$ \\
\hline No. of patients & 75 & 197 & 115 & 20 \\
\hline Country & UK & $\begin{array}{l}\text { Germany, Austria, } \\
\text { Switzerland }\end{array}$ & $\begin{array}{l}\text { Germany, Austria, } \\
\text { Switzerland }\end{array}$ & Denmark \\
\hline Male sex & $56 \%$ & $68 \%$ & - & $65 \%$ \\
\hline Concomitant allergic diseases & $85 \%$ & - & $78 \%$ & $65 \%$ \\
\hline All cofactors & $15.3 \%$ & $18.3 \%$ & $14 \%$ & $40 \%$ \\
\hline Exercise & $2.5 \%$ & $10 \%$ & $8.7 \%$ & $15 \%$ \\
\hline Infection & $2.5 \%$ & $3 \%$ & $2.6 \%$ & $15 \%$ \\
\hline NSAIDs and other drugs & - & $6 \%$ & $2.6 \%$ & $10 \%$ \\
\hline Stress, menstruation, and others & $10.3 \%$ & $\begin{array}{c}12 \% \text { (menstruation } 10 \% \\
\text { in female, Psychological } \\
\text { stress } 2 \% \text { ) }\end{array}$ & - & - \\
\hline Alcohol & - & $0 \%$ & - & $0 \%$ \\
\hline
\end{tabular}

Table 3. Proposed mechanisms of action of common cofactors

\begin{tabular}{l} 
Proposed major action mechanisms \\
\hline 1. Increased bioavailability of allergen \\
- Increased intestinal permeability and intestinal allergen absorption \\
- Increased blood circulation and influx of allergens \\
2. Decreased activation threshold on the cellular level \\
- Direct impact on mast cell and basophil activation \\
3. Transient plasma hyperosmolality \\
4. Others \\
- Increased the serum IgE concentration \\
IgE, immunoglobulin E.
\end{tabular}

with severe food allergies ( $10 \%$ vs. $16 \%, P=0.037)$. In this study, two-thirds of the patients did not know if a cofactor influenced their allergic reaction. A retrospective analysis of patients with food anaphylaxis showed that cofactors were involved in $21 \%$; among them, exercise was the most frequently implicated cofactor, followed by alcohol. ${ }^{15)}$

Among exercise-induced anaphylaxis syndrome, FDEIA is the best-studied model of cofactor-induced anaphylaxis. The food ingested 4 hours before exercise and up to 1 hour after exercise was related to FDEIA. FDEIA events are most commonly associated with jogging. However, only moderate-intensity exercise is sufficient to elicit symptoms. ${ }^{17)}$ Exercise can reportedly also augment the risk of other cofactors such as NSAID use and infection. ${ }^{18,19)}$

In oral challenge tests performed in 71 patients with WDEIA, a reaction could be elicited at rest in $37 \%$, but treadmill exercise considerably increased the severity and lowered the threshold (median dose of gluten at rest: $48 \mathrm{~g}$; median dose combined with exercise: $24 \mathrm{~g}$ ). ${ }^{20)}$

Despite recent advances, the mechanism of FDEIA is not fully understood. A few mechanisms have been suggested.

\section{Increased intestinal allergen absorption}

Exercise increases intestinal permeability and induces an increase in intestinal allergen absorption. ${ }^{21)}$ Several experimental and animal studies have demonstrated that exercise increases intestinal permeability; some suggested its mechanisms. ${ }^{22}$ Intense exercise may increase the core temperature and result in transient intestinal epithelial cell damage. In addition, the deviation of blood flow away from the splanchnic arteries to the working muscle results in gut underperfusion and ischemia, which causes epithelial damage. ${ }^{23)}$ In summary, the intestinal epithelial damage induced by the increased core temperature and gut ischemia damages tight junction integrity and increases intestinal permeability to food allergens. Acute exercise accelerated gastrointestinal leakage of lysozyme in lysozyme-sensitized but not nonsensitized mice. $^{22)}$

Serum gliadin monitoring during challenge testing was useful for identifying patients with false-negative results in WDEIA challenge tests. ${ }^{24)}$ However, a recent study ${ }^{25)}$ showed that exercise does not fundamentally increase serum peak gliadin levels in healthy volunteers. The level of zonulin, the intestinal barrier function protein, in the stool was not associated with exercise or peak gliadin concentrations. This suggests that exercise alone may not be sufficient to increase intestinal permeability and cause anaphylaxis; rather, it is likely that other combined factors such as the susceptibility to increased intestinal mucosal damage induced by exercise may be needed.

The gut microbiota is involved in intestinal barrier function ${ }^{26,27)}$; therefore, it may be a combined factor related to the increase in intestinal permeability during exercise. Butyrate, a short-chain fatty acid produced by bacterial fermentation of undigested carbohydrate in the intestine, enhances intestinal barrier function by facilitating tight junction assembly. ${ }^{28)}$ Changes in gut microbiota composition may enable cofactors such as exercise to further increase intestinal absorption. ${ }^{25)}$ The gut microbiome composi- 
tions of patients with WDEIA differed from those of healthy controls. $^{29)}$

\section{Decreased mast cell and basophil activation threshold}

Another hypothesis is that exercise directly affects mast cells and basophils and modifies the threshold dose of food allergens. ${ }^{30)}$ Physical exercise may increase plasma osmolarity, resulting in mast cell and basophil activation. However, only strenuous exercise increases plasma osmolarity, and the exercise intensity of patients with WDEIA is not usually strenuous.

\section{Activation of tissue transglutaminase}

In WDEIA, activation of tissue transglutaminase (tTG) during exercise was postulated to create large $\omega-5$ gliadin/tTG complexes that elicit anaphylactic reactions.

\section{Acute infections}

Epidemiological studies reported that infections are associated with $2.5 \%-3 \%$ of anaphylactic reactions in children. ${ }^{5,8,9)}$ In the clinical setting, infection is a commonly suspected augmentation factor for anaphylaxis. A possible association between infection and anaphylactic reactions following allergen immunotherapy is often reported. During oral tolerance induction in patients with food allergies, many unexpected symptomatic episodes occur during an infection. ${ }^{31)}$ Therefore, patients undergoing oral immunotherapy may need to decrease the dosage of protein consumed during an infection.

The action may occur mainly due to fever and subsequent increased blood circulation and the influx of allergens. In addition, gastrointestinal infections may lead to larger food proteins passing through the inflamed mucosa. In contrast to other cofactors, this cannot be confirmed via provocation tests. For this reason, how viral or bacterial infections act as cofactors has not yet been established.

Because infections are much more common in children, clinicians should consider infection as an important cofactor for food allergies and anaphylaxis, especially in children.

\section{Drugs}

\section{Nonsteroidal anti-inflammatory drugs}

NSAID use is the most common cofactor of food allergies and anaphylaxis. NSAID use is involved in up to $25 \%$ of food-induced anaphylaxis cases, representing a risk factor with an odds ratio higher than $11{ }^{18)}$ Similar to exercise, two mechanisms are sug. gested: upregulation of intestinal absorption of antigens by NSAID use and a direct impact on mast cell and basophil activation. ${ }^{14,32)}$ Prostaglandins play an important role in defending the gastrointestinal mucosa, and the increase in gastrointestinal permeability may be due to the direct cyclooxygenase (COX) pathway. The basophil activation effect of NSAIDs in food-in- duced allergic reactions might be related to the COX pathway. ${ }^{32)}$ In contrast, aspirin enhanced histamine release from basophils only when the immunoglobulin $\mathrm{E}$ ( $\mathrm{IgE}$ ) receptor signaling path. way was activated, indicating that it was not due to $\mathrm{COX}$ inhibition. ${ }^{33)}$

In 1995-2008, a case-control study was conducted to determine the role of drugs as risk factors for severe food allergy in adults. ${ }^{18)}$ In this study, aspirin, NSAID, beta-blocker, and angiotensin-converting enzyme inhibitor use were found to be substantial risk factors for IgE-dependent food allergies. Aspirin and NSAID use were associated with $15.8 \%$ and $6.6 \%$ of severe food anaphylaxis cases, respectively, and $1.7 \%$ and $0.9 \%$ of mild to moderate food allergy cases, respectively. The respective odds ratios were 10.8 and 8.2. In addition, this study showed that exercise and alcohol intake drastically increased the risk of drugs in adults with severe food allergies.

NSAID use has also been demonstrated to exacerbate food allergies in patients with FDEIA. ${ }^{34)} \mathrm{A}$ study on patients with WDEIA demonstrated that serum gliadin levels increased 5 -fold 30 min after provocation tests with wheat-aspirin challenge versus aspirin-only challenge. ${ }^{14)}$ The synergistic effect of NSAID use and food allergy is well known. Approximately one-third of patients with plant-food allergies related to lipid transfer proteins were cofactor-dependent (mainly related to NSAID use). ${ }^{35)}$ Fooddependent NSAID-induced anaphylaxis (FDNIA) is a phenotype of anaphylaxis in which NSAID use is the only cofactor. ${ }^{36}$ In the case of FDNIA, a provocation test with the culprit drug would be negative if the food allergen is not present. ${ }^{19)}$

Atopy is a risk factor for hypersensitivity reactions to NSAID use. In atopic adolescents, isolated periorbital angioedema was the most frequent manifestation of NSAID intolerance. ${ }^{37)}$ The role of aspirin and NSAID use as cofactors has not been fully evaluated in children and adolescents. However, NSAID use must be considered a cofactor, especially in adolescents with severe food allergies. Along with exercise, NSAID use synergistically increases intestinal barrier permeability. Patients with severe food allergies may need to avoid aspirin and NSAID use for $24-48$ hours prior to exercising.

\section{H2-receptor antagonists, proton pump inhibitors, and beta- adrenoceptor antagonists}

Gastric acid modulates or inactivates protein allergens by digesting H2-receptor antagonists and proton pump inhibitors (PPIs), which interfere with these functions by increasing the gastric $\mathrm{pH}$ and may lead to less effective inactivation of food allergens and allergic reactions. Mouse studies have shown that PPI use increases the risks of food sensitization and anaphylaxis. ${ }^{38)}$ Patients with oral allergy syndrome in particular must be informed of the possible role of PPIs as a cofactor of anaphylaxis.

Beta-adrenoceptor antagonist use leads to the inhibition of beta-adrenergic signals on mast cells and basophils and consequently to the destabilization of these effector cells of anaphylaxis. Moreover, beta-adrenoceptor antagonists inhibit blood pressureregulating mechanisms and facilitate anaphylaxis induction and 
severity. In adult patients with anaphylaxis, antihypertensive medication use, including beta-blockers, is associated with increased organ system involvement and hospitalization. ${ }^{39)}$

\section{Alcohol}

Alcohol is reportedly a cofactor of anaphylaxis in 1.0\%$15.2 \%$ of patients. ${ }^{7)}$ A questionnaire study showed that alcohol is the second most important cofactor in food-related allergic reactions in patients aged $\geq 16$ years with food allergies. ${ }^{2)}$ Although the mechanisms of this phenomenon are not well established, increased intestinal absorption seems to be involved. Christensen et al. ${ }^{16}$ performed an oral challenge with gluten at rest and combined it with treadmill exercise, aspirin, alcohol, or combined exercise and aspirin in 25 adult patients with WDEIA. This study demonstrated that exercise and aspirin augment clinical reactions in WDEIA by lowering the threshold and increasing the severity of the allergic reaction, whereas alcohol functioned only as a cofactor in a minority of the patients, sug. gesting that it is a weak cofactor.

\section{Stress and sleep deprivation}

Allergic diseases such as asthma and atopic dermatitis can be exacerbated because of acute stress related to the release of neuropeptides and neurotransmitters in the central nervous system, which activate the allergic inflammatory response. ${ }^{40)}$ Food allergy outcomes can also be affected by psychosocial stress. ${ }^{41)}$ However, the role of psychosocial stress in food-related allergic reactions has been ignored until recently.

In animal studies, increased intestinal permeability was identified as the link between acute psychological stress and an increased mucosal immune response via enhanced antigen penetration. ${ }^{42,43)} \mathrm{A}$ few human studies demonstrated that acute stress increases small intestinal permeability. ${ }^{44,45)}$ However, the effect of stress on intestinal permeability in humans remains unclear. Acute emotional stress is postulated as a risk factor for anaphylaxis in patients receiving allergen immunotherapy. ${ }^{46)}$ Regardless of whether acute stress is physical or psychological, similar responses occur in the human body. ${ }^{45}$ )

In a crossover study, ${ }^{13)}$ patients with peanut allergy underwent 3 open peanut challenges in random order: with exercise after each dose, with sleep deprivation preceding the challenge, and with no intervention. For the first time, this study showed that, along with exercise, sleep deprivation markedly reduced the threshold dose, triggering symptoms by $45 \%$. No study to date has examined the role of sleep deprivation in food-allergic reactions. Although the mechanism is unknown, sleep deprivation may stress the gastrointestinal tract, thereby enhancing intestinal per. meability.

\section{Dehydration}

Dehydration directly or indirectly impairs stroke volume, cardiac output, and skin blood flow, resulting in larger increases in core temperature and heart rate. ${ }^{47)}$ Acute airway dehydration is reportedly a trigger for bronchoconstriction in exerciseinduced asthma. ${ }^{48)}$ Inadequate fluid intake before and/or during exercise may augment exercise-induced airway dehydration. Dehydration may be an important factor in allergic responses. However, few studies have analyzed the effects of dehydration on food allergies and anaphylaxis. In rats, water avoidance stress for 10 days decreased intestinal epithelial tight junction proteins such as occludin and claudin- $1{ }^{49)}$ The role of dehydrationinduced gut damage was a postulated mechanism. ${ }^{50)}$ On the contrary, using an experiment of cycling exercise in dehydrated and euhydrated conditions, van Nieuwenhoven et al. ${ }^{51)}$ showed that dehydration did not lead to differences in intestinal permeability in healthy men. Although the role of dehydration in food-allergic reactions requires evaluation, dehydration may augment the effect of exercise in patients with FDEIA.

\section{Conclusions}

The phenomenon of cofactors is well known but frequently neglected. Cofactors such as exercise, infections, NSAID use, dehydration, and alcohol intake can increase intestinal permeability and result in increased antigen uptake, thereby causing allergic symptoms.

Along with identifying the eliciting allergen, routine assessments of the possible involvement of cofactors are essential for the management of patients with food-induced anaphylaxis to avoid life-threatening anaphylactic episodes. In patients with a suggestive history but a negative challenge test result, the possible involvement of cofactors should be considered. If possible, oral food challenges combined with a suspected cofactor should be performed more actively.

\section{Footnotes}

Conflict of interest: No potential conflicts of interest relevant to this article are reported.

Acknowledgments: This work was supported by the Soonchunhyang University Research Fund.

\section{References}

1. Lee SY, Ahn K, Kim J, Jang GC, Min TK, Yang HJ, et al. A multicenter retrospective case study of anaphylaxis triggers by age in Korean children. Allergy Asthma Immunol Res 2016;8:535-40.

2. Huang F, Chawla K, Jarvinen KM, Nowak-Wegrzyn A. Anaphylaxis in a New York City pediatric emergency department: triggers, treatments, and 
outcomes. J Allergy Clin Immunol 2012;129:162-8.e1-3.

3. Muraro A, Roberts G, Worm M, Bilo MB, Brockow K, Fernandez Rivas $\mathrm{M}$, et al. Anaphylaxis: guidelines from the European Academy of Allergy and Clinical Immunology. Allergy 2014;69:1026-45.

4. Niggemann B, Beyer K. Factors augmenting allergic reactions. Allergy 2014;69:1582-7.

5. Worm M, Scherer K, Kohli-Wiesner A, Rueff F, Mahler V, Lange L, et al. Food-induced anaphylaxis and cofactors - data from the anaphylaxis registry. Allergol Select 2017;1:21-7.

6. Cardona V, Luengo O, Garriga T, Labrador-Horrillo M, Sala-Cunill A, Izquierdo A, et al. Co-factor-enhanced food allergy. Allergy 2012;67: 1316-8.

7. Wolbing F, Fischer J, Koberle M, Kaesler S, Biedermann T. About the role and underlying mechanisms of cofactors in anaphylaxis. Allergy 2013;68: 1085-92.

8. Uguz A, Lack G, Pumphrey R, Ewan P, Warner J, Dick J, et al. Allergic reactions in the community: a questionnaire survey of members of the anaphylaxis campaign. Clin Exp Allergy 2005;35:746-50.

9. Hompes S, Kohli A, Nemat K, Scherer K, Lange L, Rueff F, et al. Provoking allergens and treatment of anaphylaxis in children and adolescents--data from the anaphylaxis registry of German-speaking countries. Pediatr Allergy Immunol 2011;22:568-74.

10. Oropeza AR, Bindslev-Jensen C, Broesby-Olsen S, Kristensen T, Møller $\mathrm{MB}$, Vestergaard $\mathrm{H}$, et al. Patterns of anaphylaxis after diagnostic workup: A follow-up study of 226 patients with suspected anaphylaxis. Allergy 2017;72:1944-52.

11. Versluis A, van Os-Medendorp H, Blom WM, Michelsen-Huisman AD, Castenmiller JJM, Noteborn $\mathrm{H}$, et al. Potential cofactors in accidental food allergic reactions are frequently present but may not influence severity and occurrence. Clin Exp Allergy 2019;49:207-15.

12. Versluis A, van Os-Medendorp H, Kruizinga AG, Blom WM, Houben GF, Knulst AC. Cofactors in allergic reactions to food: physical exercise and alcohol are the most important. Immun Inflamm Dis 2016:4:392-400.

13. Dua S, Ruiz-Garcia M, Bond S, Durham SR, Kimber I, Mills C, et al. Effect of sleep deprivation and exercise on reaction threshold in adults with peanut allergy: a randomized controlled study. J Allergy Clin Immunol 2019;144:1584-94.e2.

14. Matsuo H, Morimoto K, Akaki T, Kaneko S, Kusatake K, Kuroda T, et al. Exercise and aspirin increase levels of circulating gliadin peptides in patients with wheat-dependent exercise-induced anaphylaxis. Clin Exp Allergy 2005;35:461-6.

15. Fernandes RA, Regateiro F, Pereira C, Faria E, Pita J, Todo-Bom A, et al. Anaphylaxis in a food allergy outpatient department: one-year review. Eur Ann Allergy Clin Immunol 2018;50:81-8.

16. Christensen MJ, Eller E, Mortz CG, Brockow K, Bindslev-Jensen C. A multicenter retrospective case study of anaphylaxis triggers by age in Korean children. J Allergy Clin Immunol Pract 2019;7:114-21.

17. Barg W, Medrala W, Wolanczyk-Medrala A. Exercise-induced anaphylaxis: an update on diagnosis and treatment. Curr Allergy Asthma Rep 2011;11: 45-51.

18. Moneret-Vautrin DA, Latarche C. Drugs as risk factors of food anaphylaxis in adults: a case-control study. Bull Acad Natl Med 2009;193:351-62; discussion 62-3.

19. Bartra J, Araujo G, Munoz-Cano R. Interaction between foods and nonsteroidal anti-inflammatory drugs and exercise in the induction of anaphy. laxis. Curr Opin Allergy Clin Immunol 2018;18:310-6.

20. Christensen MJ, Eller E, Mortz CG, Brockow K, Bindslev-Jensen C. Exercise lowers threshold and increases severity, but wheat-dependent, exercise-induced anaphylaxis can be elicited at rest. J Allergy Clin Immunol Pract 2018;6:514-20.

21. Karhu E, Forsgard RA, Alanko L, Alfthan H, Pussinen P, Hamalainen E, et al. Exercise and gastrointestinal symptoms: running-induced changes in intestinal permeability and markers of gastrointestinal function in asymptomatic and symptomatic runners. Eur J Appl Physiol 2017;117:2519-26.

22. Yano $\mathrm{H}$, Kato $\mathrm{Y}$, Matsuda T. Acute exercise induces gastrointestinal leakage of allergen in lysozyme-sensitized mice. Eur J Appl Physiol 2002;87: $358-64$.
23. Zuhl M, Schneider S, Lanphere K, Conn C, Dokladny K, Moseley P. Exercise regulation of intestinal tight junction proteins. Br J Sports Med 2014;48:980-6.

24. Kohno K, Matsuo H, Takahashi H, Niihara H, Chinuki Y, Kaneko S, et al. Serum gliadin monitoring extracts patients with false negative results in challenge tests for the diagnosis of wheat-dependent exercise-induced anaphylaxis. Allergol Int 2013;62:229-38.

25. Scherf KA, Lindenau AC, Valentini L, Collado MC, Garcia-Mantrana I, Christensen M, et al. Cofactors of wheat-dependent exercise-induced anaphylaxis do not increase highly individual gliadin absorption in healthy volunteers. Clin Transl Allergy 2019;9:19.

26. Alam A, Neish A. Role of gut microbiota in intestinal wound healing and barrier function. Tissue Barriers 2018;6:1539595.

27. Takiishi T, Fenero CIM, Camara NOS. Intestinal barrier and gut microbiota: Shaping our immune responses throughout life. Tissue Barriers 2017;5:e1373208.

28. Peng L, Li ZR, Green RS, Holzman IR, Lin J. Butyrate enhances the in testinal barrier by facilitating tight junction assembly via activation of AMP-activated protein kinase in Caco-2 cell monolayers. J Nutr 2009; 139:1619-25.

29. Du Z, Gao X, Yin J. Gut microbiome alterations in patients with wheatdependent exercise-induced anaphylaxis. Int Immunopharmacol 2020; 84:106557.

30. Barg W, Wolanczyk-Medrala A, Obojski A, Wytrychowski K, Panaszek B, Medrala W. Food-dependent exercise-induced anaphylaxis: possible impact of increased basophil histamine releasability in hyperosmolar conditions. J Investig Allergol Clin Immunol 2008;18:312-5.

31. Staden U, Rolinck-Werninghaus C, Brewe F, Wahn U, Niggemann B, Beyer K. Specific oral tolerance induction in food allergy in children: efficacy and clinical patterns of reaction. Allergy 2007;62:1261-9.

32. Pascal M, Munoz-Cano R, Mila J, Sanz ML, Diaz-Perales A, SanchezLopez J, et al. Nonsteroidal anti-inflammatory drugs enhance IgE-mediated activation of human basophils in patients with food anaphylaxis dependent on and independent of nonsteroidal anti-inflammatory drugs. Clin Exp Allergy 2016;46:1111-9.

33. Matsuo H, Yokooji T, Morita H, Ooi M, Urata K, Ishii K, et al. Aspirin aug ments IgE-mediated histamine release from human peripheral basophils via Syk kinase activation. Allergol Int 2013;62:503-11.

34. Harada S, Horikawa T, Ashida M, Kamo T, Nishioka E, Ichihashi M. Aspirin enhances the induction of type I allergic symptoms when combined with food and exercise in patients with food-dependent exercise-induced anaphylaxis. Br J Dermatol 2001;145:336-9.

35. Pascal M, Muñoz-Cano R, Reina Z, Palacín A, Vilella R, Picado C, et al. Lipid transfer protein syndrome: clinical pattern, cofactor effect and profile of molecular sensitization to plant-foods and pollens. Clin Exp Allergy 2012;42:1529-39.

36. Dona I, Perez-Sanchez N, Eguiluz-Gracia I, Munoz-Cano R, Bartra J, Torres MJ, et al. Progress in understanding hypersensitivity reactions to nonsteroidal anti-inflammatory drugs. Allergy 2020;75:561-75.

37. Quiralte J, Blanco C, Castillo R, Delgado J, Carrillo T. Intolerance to nonsteroidal antiinflammatory drugs: results of controlled drug challenges in 98 patients. J Allergy Clin Immunol 1996;98:678-85.

38. Diesner SC, Knittelfelder R, Krishnamurthy D, Pali-Scholl I, Gajdzik L, Jensen-Jarolim E, et al. Dose-dependent food allergy induction against ovalbumin under acid-suppression: a murine food allergy model. Immunol Lett 2008;121:45-51.

39. Lee S, Hess EP, Nestler DM, Bellamkonda Athmaram VR, Bellolio MF, Decker WW, et al. Antihypertensive medication use is associated with increased organ system involvement and hospitalization in emergency department patients with anaphylaxis. J Allergy Clin Immunol 2013;131: 1103-8.

40. Dave ND, Xiang L, Rehm KE, Marshall GD, Jr. Stress and allergic diseases. Immunol Allergy Clin North Am 2011;31:55-68.

41. Schreier HM, Wright RJ. Stress and food allergy: mechanistic considerations. Ann Allergy Asthma Immunol 2014;112:296-301.

42. Agostini S, Goubern M, Tondereau V, Salvador-Cartier C, Bezirard V, Leveque $\mathrm{M}$, et al. A marketed fermented dairy product containing Bifido- 
bacterium lactis $\mathrm{CNCM}$-2494 suppresses gut hypersensitivity and colonic barrier disruption induced by acute stress in rats. Neurogastroenterol Motil 2012;24:376-e172.

43. Saunders PR, Kosecka U, McKay DM, Perdue MH. Acute stressors stimulate ion secretion and increase epithelial permeability in rat intestine. Am J Physiol 1994;267:G794-9.

44. Vanuytsel T, van Wanrooy S, Vanheel H, Vanormelingen C, Verschueren S, Houben E, et al. Psychological stress and corticotropin-releasing hormone increase intestinal permeability in humans by a mast cell-dependent mechanism. Gut 2014;63:1293-9.

45. Alonso C, Guilarte M, Vicario M, Ramos L, Rezzi S, Martinez C, et al. Acute experimental stress evokes a differential gender-determined increase in human intestinal macromolecular permeability. Neurogastroenterol Motil 2012;24:740-6, e348-9.

46. Larenas-Linnemann DE, Costa-Dominguez MDC, Creticos PS. Acute emotional stress proposed as a risk factor for anaphylaxis in patients receiving allergen immunotherapy. Ann Allergy Asthma Immunol 2020;124: 314-7.

47. Coyle EF, Montain SJ. Benefits of fluid replacement with carbohydrate during exercise. Med Sci Sports Exerc 1992;24:S324-30.
48. Anderson SD, Kippelen P. Airway injury as a mechanism for exerciseinduced bronchoconstriction in elite athletes. J Allergy Clin Immunol 2008;122:225-35; quiz 36-7.

49. Zong Y, Zhu S, Zhang S, Zheng G, Wiley JW, Hong S. Chronic stress and intestinal permeability: Lubiprostone regulates glucocorticoid receptormediated changes in colon epithelial tight junction proteins, barrier function, and visceral pain in the rodent and human. Neurogastroenterol Motil 2019;31:e13477.

50. Kim KB, Kwak YS. Dehydration affects exercise-induced asthma and anaphylaxis. J Exerc Rehabil 2019;15:647-50.

51. van Nieuwenhoven MA, Vriens BE, Brummer RJ, Brouns F. Effect of dehydration on gastrointestinal function at rest and during exercise in humans. Eur J Appl Physiol 2000;83:578-84.

How to cite this article: Shin M. Food allergies and foodinduced anaphylaxis: role of cofactors. Clin Exp Pediatr 2021; 64:393-9. https://doi.org/10.3345/cep.2020.01088 